\title{
Photodynamic Therapy using Talaporfin Sodium for the Recurrence of Cholangiocarcinoma after Surgical Resection
}

\author{
Shuya Shimizu ${ }^{1}$, Takahiro Nakazawa ${ }^{1}$, Kazuki Hayashi ${ }^{1}$, Itaru Naitoh ${ }^{1}$, Katsuyuki Miyabe ${ }^{1}$, \\ Hiromu Kondo ${ }^{1}$, Yuji Nishi ${ }^{1}$, Shuichiro Umemura ${ }^{1}$, Yasuki Hori ${ }^{1}$, Akihisa Kato ${ }^{1}$, \\ Tomohito Morisaki ${ }^{2}$, Atsushi Nanashima ${ }^{3}$, Hirotaka Ohara ${ }^{4}$ and Takashi Joh ${ }^{1}$
}

\begin{abstract}
We herein report the case of a 72-year-old man who underwent photodynamic therapy (PDT) with talaporfin sodium for recurrent cholangiocarcinoma after surgical resection. Endoscopic retrograde cholangiography (ERC) showed severe stenosis with an irregular surface measuring approximately $1 \mathrm{~cm}$ in length from the anastomotic site, and a recurrent nodular lesion was observed at the anastomotic site of the right anterior intrahepatic bile duct on gastrointestinal endoscopy. ERC after PDT revealed a dramatic improvement in the bile duct stenosis, and the nodular lesion had disappeared. No adverse events from the PDT were detected. PDT using talaporfin sodium may be a safe alternative treatment for cholangiocarcinoma.
\end{abstract}

Key words: photodynamic therapy, talaporfin sodium, Laserphyrin ${ }^{\circledR}$, PD laser ${ }^{\circledR}$, cholangiocarcinoma

(Intern Med 54: 2321-2326, 2015)

(DOI: 10.2169/internalmedicine.54.4673)

\section{Introduction}

Photodynamic therapy (PDT) is a local therapy used to treat cholangiocarcinomas which consists of the intravenous administration of a photosensitizing agent, its accumulation in the target tumor tissue, and subsequent illumination with laser light. Irradiation with light of an appropriate wavelength activates the photosensitizer, which transfers energy to oxygen, triggering a series of reactions leading to cell apoptosis or necrosis. PDT has been used to treat various tumors and premalignant tissues (e.g., bladder carcinoma, gastric carcinoma, and Barrett's esophagus with dysplasia) (1). Although several types of photosensitizing drugs, such as porfimer sodium (Photofrin ${ }^{\circledR}$, Pfizer Japan, Tokyo, Japan) and temoporfin (mTHPC; Foscan ${ }^{\circledR}$, Biolitec AG, Jena, Germany), have been used in PDT for cholangiocarcinoma, few studies of PDT using talaporfin sodium (Laserphyrin ${ }^{\circledR}$, Meiji Seika Pharma, Tokyo, Japan) for cholangiocarcinoma have been conducted (2).

First-generation PDT using porfimer sodium as a photosensitizer has a high risk of skin phototoxicity and requires a relatively long sun-shade period (4-6 weeks) and a large and expensive excimer dye laser system. In contrast, talaporfin sodium in second-generation PDT is cleared more rapidly from the skin compared with porfimer sodium. It is also theoretically expected to have a lower rate of phototoxicity with a shorter sun-shade period ( 2 weeks). Furthermore, talaporfin sodium is typically administered 6 hours before laser light illumination, in contrast to 48 hours before for porfimer sodium, and the diode laser system (PD laser ${ }^{\circledR}$, Panasonic Healthcare, Toon, Japan) that excites talaporfin sodium is smaller than the excimer dye laser system. Therefore, PDT using talaporfin sodium is easier to perform. However, its clinical efficacy and safety for cholangiocarcinoma has not yet been fully evaluated, as PDT with talaporfin sodium and PD laser ${ }^{\mathbb{R}}$ is approved only for early lung cancer under the Japanese health insurance system.

\footnotetext{
${ }^{1}$ Department of Gastroenterology and Metabolism, Nagoya City University Graduate School of Medical Sciences, Japan, ${ }^{2}$ Department of Gastroenterology and Hepatology, National Hospital Organization Ureshino Medical Center, Japan, ${ }^{3}$ Department of Surgical Oncology, Nagasaki University Graduate School of Biomedical Sciences, Japan and ${ }^{4}$ Department of Community-based Medical Education, Nagoya City University Graduate School of Medical Sciences, Japan

Received for publication December 8, 2014; Accepted for publication January 15, 2015

Correspondence to Dr. Takahiro Nakazawa, tnakazaw@med.nagoya-cu.ac.jp
} 


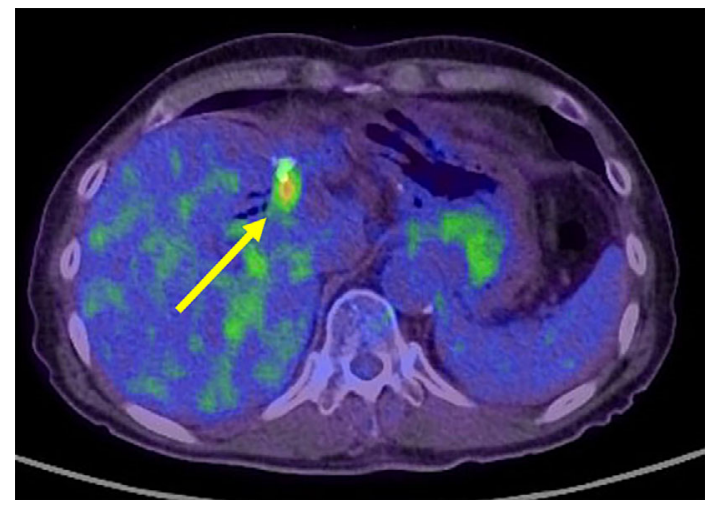

Figure 1. Positron emission tomography/computed tomography revealed a positive ${ }^{18} \mathrm{~F}$-fluorodeoxyglucose uptake at the hepatic hilar area (arrow).

The curative therapy for cholangiocarcinoma is surgery. However, surgery is invasive, and tumors may be unresectable at the time of diagnosis. Therefore, increasing reports utilizing PDT to treat cholangiocarcinoma have recently been published. PDT for cholangiocarcinoma was performed for purposes of palliative management in many cases (3-8), and cases with a long-term survival without recurrence have been reported. As PDT induces necrosis to tissue at a depth of only 4-6 $\mathrm{mm}$, it cannot provide a cure for invasive cholangiocarcinoma. However, it is possible to achieve a complete cure for the superficial spreading type of cholangiocarcinoma because the lesion depth is relatively shallow.

We herein describe the use of PDT with talaporfin sodium and PD laser ${ }^{\circledR}$ as a possible curative treatment for the recurrent superficial spreading type of cholangiocarcinoma after surgical resection.

\section{Case Report}

A 72-year-old man underwent a pancreatoduodenectomy and extrahepatic bile duct excision combined with hilar ductoplasty and hepaticojejunostomy for the superficial spreading type of lower bile duct carcinoma in 2008. He was diagnosed in 2012 with recurrent cholangiocarcinoma at the anastomotic site of the left hepatic duct and received a left hepatic lobectomy and partial jejunectomy. However, positron emission tomography / CT imaging with ${ }^{18} \mathrm{~F}$ fluorodeoxyglucose (FDG) in April 2014 confirmed a positive FDG uptake at the hepatic hilar area (Fig. 1). Therefore, he was admitted to our hospital in June 2014 for further examination due to suspected recurrence of cholangiocarcinoma.

The patient exhibited no symptoms on admission, and his physical examination revealed no abnormal findings with the exception of fever. The laboratory data showed elevated serum levels of aspartate aminotransferase (44 U/L; normal range, 13-33 U/L), alanine aminotransferase (35 U/L; normal range, 6-30 U/L), and $\gamma$-glutamyl transpeptidase (75 U/ $\mathrm{L}$; normal range, 10-47 U/L). The serum C-reactive protein level was $10.33 \mathrm{mg} / \mathrm{dL}$ (normal range, $\leq 0.3 \mathrm{mg} / \mathrm{dL}$ ), and the WBC count was $12,500 / \mathrm{mm}^{3}$ (normal range, 3,600-9,600/ $\mathrm{mm}^{3}$ ). A contrast-enhanced CT scan showed a low-density $1-\mathrm{cm}$ mass at the hepatic hilum, and the right anterior intrahepatic bile duct was slightly dilated. Based on these findings, he was diagnosed with cholangitis, which improved after administration of antibiotics. After the amelioration of cholangitis, the serum levels of carcinoembryonic antigen and cancer antigen 19-9 were $1.1 \mathrm{ng} / \mathrm{mL}$ (normal range, $\leq$ $5.0 \mathrm{ng} / \mathrm{mL}$ ) and $49.8 \mathrm{U} / \mathrm{mL}$ (normal range, $\leq 37.0 \mathrm{U} / \mathrm{mL}$ ), respectively. Endoscopic retrograde cholangiography (ERC) showed severe stenosis and an irregular surface measuring approximately $1 \mathrm{~cm}$ in length at the anastomotic site (Fig. 2A). A nodular lesion was detected at the anastomotic site of the right anterior intrahepatic bile duct on endoscopy (Fig. 3A). A biopsy specimen from the nodular lesion revealed adenocarcinoma. Therefore, we histopathologically diagnosed this case as recurrent cholangiocarcinoma. We considered that the patient should undergo surgical resection as a curative therapy before PDT. However, this patient already underwent left hepatic lobectomy in 2012 and the residual liver volume was not enough to avoid fatal hepatic failure following liver resection. Therefore, he could not undergo surgical resection for recurrent cholangiocarcinoma. Surgical resection was considered to be difficult and no distant or lymph node metastases were found. Moreover, the bile duct wall thickness in the regions of the stricture on a cholangiogram was up to $2.8 \mathrm{~mm}$ according to the intraductal ultrasonography (IDUS) findings. Accordingly, we performed PDT with the approval of the Institutional Review Board of the Nagoya City University Graduate School of Medical Sciences (\#42-12-0001), and written informed consent was obtained from the patient in accordance with the Declaration of Helsinki.

The photosensitizer talaporfin sodium (Laserphyrin ${ }^{\circledR}$ ) was injected intravenously at a dose of $40 \mathrm{mg} / \mathrm{m}^{2}$ body surface area 6 hours before PDT. The patient was instructed to avoid sunlight for 2 weeks after the administration of talaporfin sodium due to persistent skin photosensitivity. A 664-nm semiconductor laser was applied by endoscopy to the tumor lesion within 6 hours after talaporfin sodium injection. Photoactivation with the diode laser system (PD la$\operatorname{ser}^{\mathbb{R}}$ ) was performed at $664 \mathrm{~nm}$ with a light dose of $100 \mathrm{~J} /$ $\mathrm{cm}^{2}$ and a fluence rate of $150 \mathrm{~mW} / \mathrm{cm}^{2}$. The stenosis was irradiated by a 1-cm-long cylindrical light diffuser (Fig. 4), and the recurrent nodular lesion at the anastomotic site of the right anterior intrahepatic bile duct was irradiated with a frontal light distributor (Fig. 5). An ischemic change was observed at the laser-irradiated lesion immediately after PDT (Fig. 3B). The elevated serum levels of aspartate aminotransferase and alanine aminotransferase of this patient before PDT showed improvement to the serum levels of 21 $\mathrm{U} / \mathrm{L}$ and $22 \mathrm{U} / \mathrm{L}$ after PDT, respectively, and no adverse events were detected during or after these procedures.

The nodular lesion disappeared 1 week after PDT, and a slightly erosive lesion was observed at the anastomotic site 

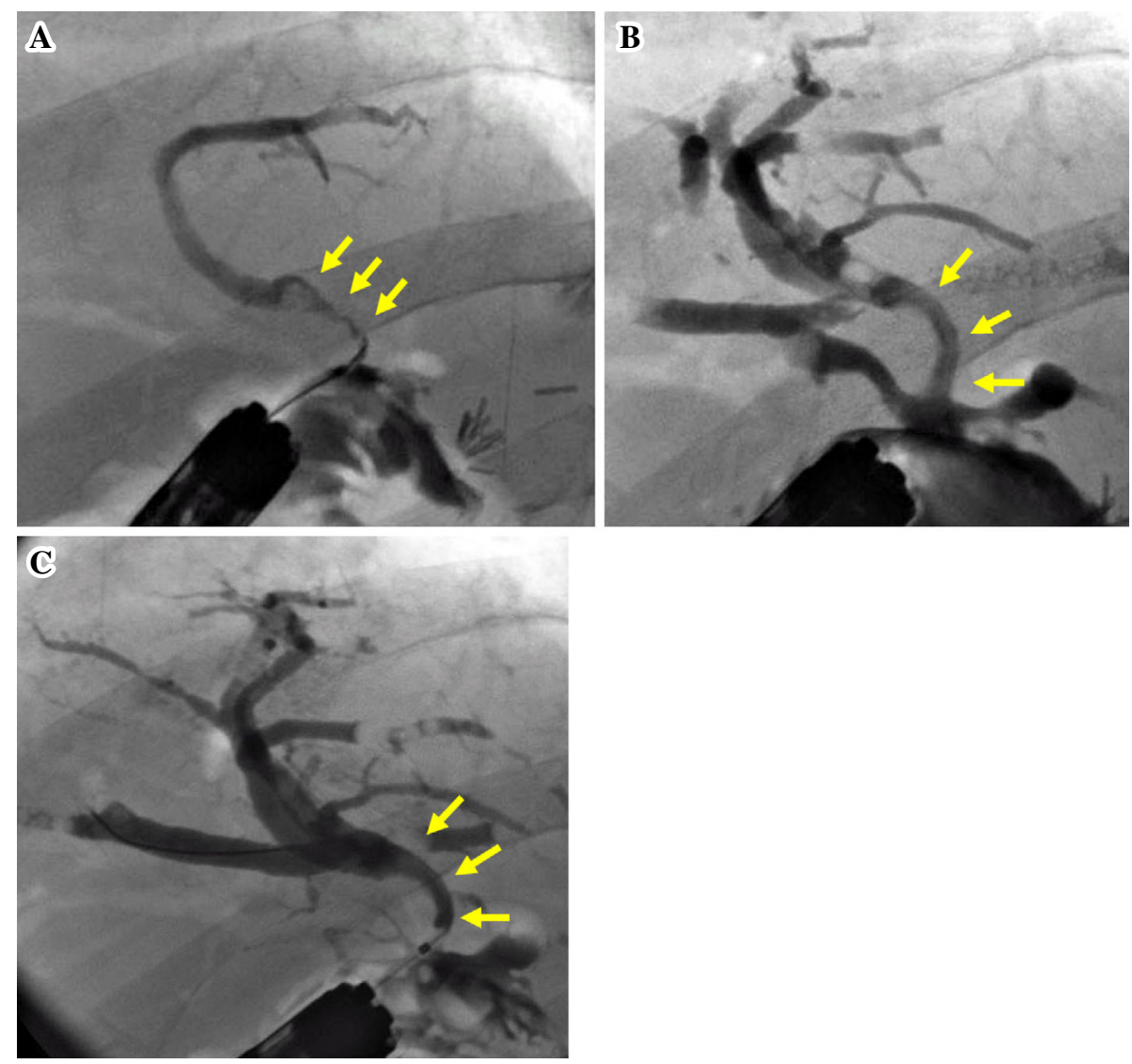

Figure 2. Endoscopic retrograde cholangiography (ERC) findings before and after photodynamic therapy (PDT). (A) Severe stenosis and an irregular surface measuring approximately $1 \mathrm{~cm}$ in length at the anastomotic site were observed before PDT (arrows). (B) ERC revealed a remarkable improvement in the bile duct stenosis after 1 week of PDT (arrows). (C) No bile duct stenosis was observed at the 1-month follow-up (arrows).

on endoscopy (Fig. 3C). ERC revealed remarkable improvement in the bile duct stenosis (Fig. 2B). Endoscopy showed a slight cicatricial stenotic change, and no residual tumor at the anastomotic site of the right anterior intrahepatic bile duct at the 1-month follow-up was observed (Fig. 3D). No bile duct stenosis was observed by ERC (Fig. 2C). The biopsy specimens from the treated bile duct lesion and the anastomotic site after the PDT procedure were not malignant. The patient was discharged, and we planned follow-up studies every 3-6 months.

\section{Discussion}

The clinical usefulness and survival benefits of PDT for unresectable cholangiocarcinoma have been previously reported $(1,3-6)$, but the use of PDT for resectable cholangiocarcinoma as neoadjuvant or adjuvant therapy is the subject of several reports (9-12). The depth of tumor necrosis caused by PDT depends on the photosensitizer and the activation at the optimal wavelength. Porfimer sodium is one of the most widely used PDT photosensitizers for cholangiocarcinoma, and a 4-6 $\mathrm{mm}$ decrease in tumor thickness has been reported (13). PDT with talaporfin sodium was expected to have a greater effect on deep-tissue layers com- pared with porfimer sodium in a previous report (14), and the superficial spreading type of cholangiocarcinoma does not typically infiltrate into deep layers (15). Therefore, PDT with talaporfin sodium is theoretically a curative treatment for non-invasive cholangiocarcinoma without distant metastasis, such as the superficial spreading type. In the present case, we decided to perform PDT with talaporfin sodium, as the wall thickness of the bile duct stricture region was up to $2.8 \mathrm{~mm}$ on IDUS. A dramatic improvement in the bile duct findings, and no adverse events, were observed in our patient. Accordingly, PDT using talaporfin sodium may be a possible curative therapy for the superficial spreading type of cholangiocarcinoma, particularly in patients at high risk for surgery, such as those undergoing hepatopancreatoduodenectomy or pancreatoduodenectomy. Even if recurrent cholangiocarcinoma occurs, PDT would be effective for palliative purposes. According to Nanashima et al. who utilized PDT with talaporfin sodium for bile duct carcinoma in 13 patients (12), five had no tumor recurrence. The authors mentioned that the cytocidal effect of PDT using talaporfin sodium was significantly increased compared with that of PDT using porfimer sodium; thus, we expected a greater efficacy in tumor necrosis with PDT using talaporfin sodium.

Damage to surrounding organs and the vascular system is 

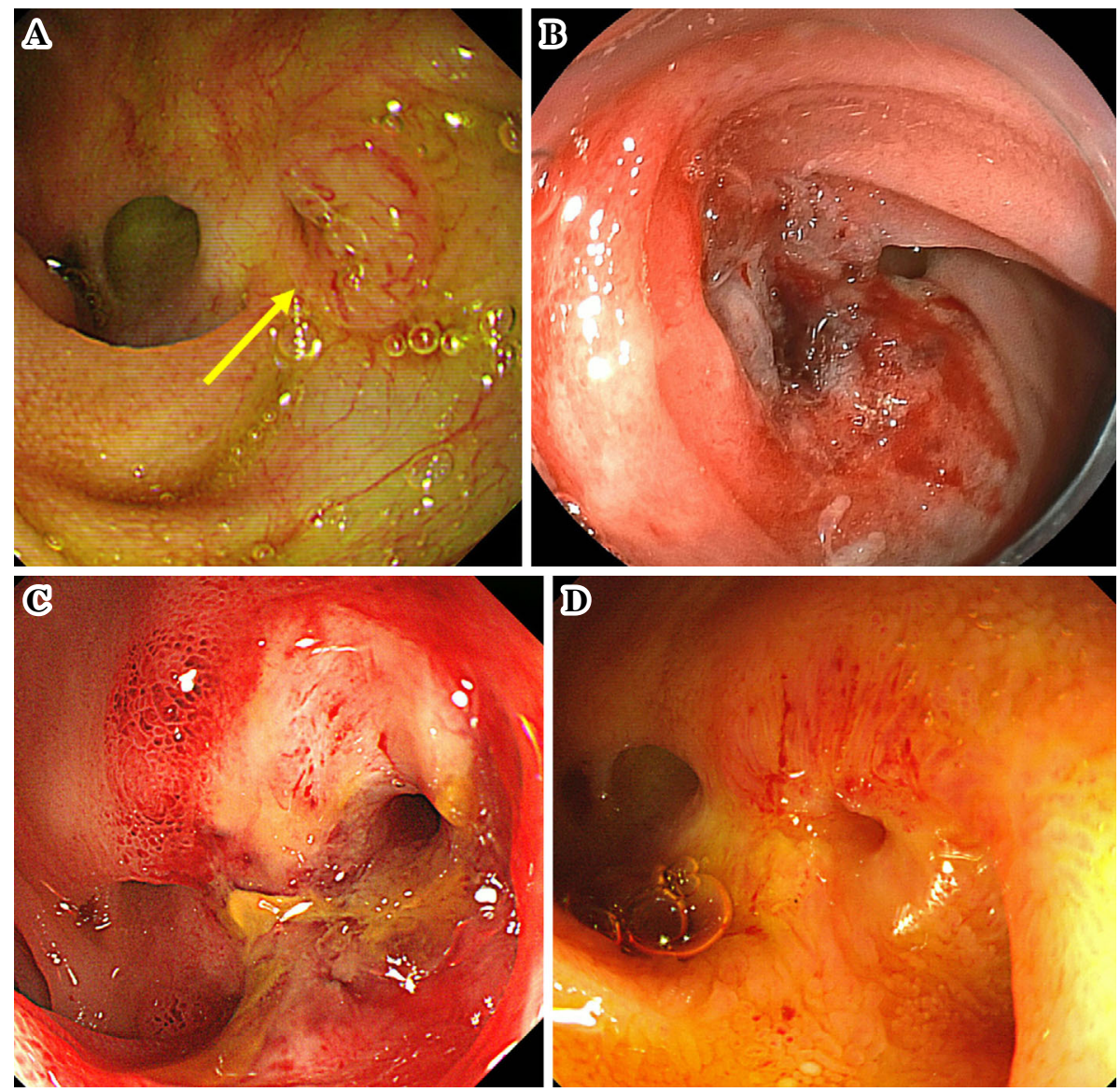

Figure 3. The endoscopic findings at the anastomotic site of right anterior intrahepatic bile duct. (A) A nodular lesion was detected at the anastomotic site of the right anterior intrahepatic bile duct before PDT (arrow). (B) An ischemic change was observed at the laser-irradiated lesion immediately after PDT. (C) The nodular lesion disappeared 1 week after PDT, and a slightly erosive lesion was observed at the anastomotic site. (D) Endoscopy showed a slight cicatricial stenotic change, and no residual tumor at the anastomotic site of the right anterior intrahepatic bile duct at the 1-month follow-up was observed.

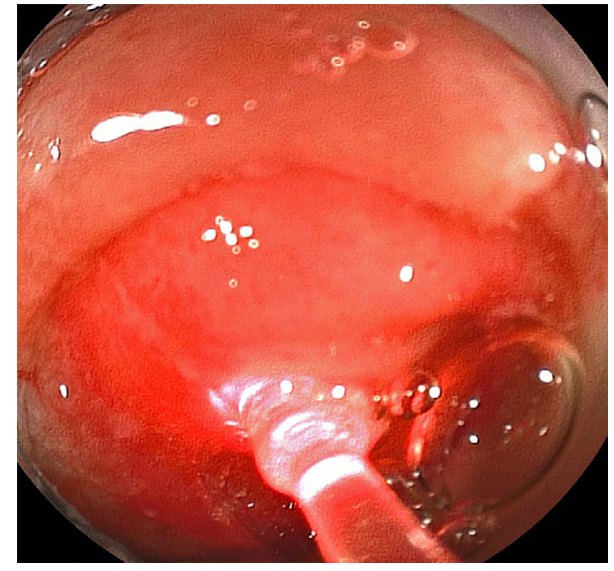

Figure 4. The stenosis was irradiated by a 1-cm-long cylindrical light diffuser.

a concern when performing PDT. Adverse events, such as cholangitis, biloma, cholecystitis, pancreatitis, sepsis, and biliary leakage have also been reported, albeit rarely (16). Nanashima et al. reported that photodermatitis, liver dys-

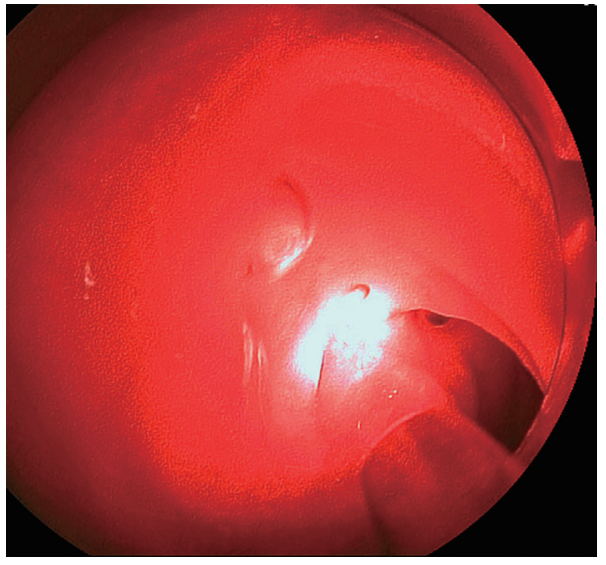

Figure 5. The recurrent nodular lesion at the anastomotic site of the right anterior intrahepatic bile duct was irradiated with a frontal light distributor.

function, cholangitis, pancreatitis, and liver abscess were each observed in at least one patient following PDT with talaporfin sodium in their study, and no endoscopy-related ad- 
verse events were observed in any patients (12). Serious adverse events have not been reported, and PDT is a generally well-tolerated treatment. Although the main portal vein was very close to the recurrent bile duct lesion in the present case, no adverse events, such as damage to the vascular system or surrounding hepatic area, occurred. Skin phototoxicity is the most common PDT adverse event; however, no dermatological events occurred in the present case after a 2week sun-shade period. According to the literature (12), patients undergoing PDT with porfimer sodium had a significantly longer admission period compared to patients undergoing PDT with talaporfin sodium (36 vs. 5 days, respectively; $\mathrm{p}<0.01)$. In the present case, the period of hospitalization was 14 days to avoid skin phototoxicity, however, this period was shorter than the reported period of PDT with porfimer sodium in the study by Nanashima et al. (12). Therefore, the efficacy, safety, and period of hospitalization with PDT using talaporfin sodium in the present case were similar to those of their report (12).

Several additional devices are needed for the PDT cholangiocarcinoma irradiation procedure. Two types of light diffuser fiber exist: a cylindrical light diffuser, which illuminates circumferentially, and a frontal light distributor, which irradiates from the front. It is possible to use the frontal light distributor under cholangioscopy for PDT of a cholangiocarcinoma. However, we typically utilized a cylindrical light diffuser under ERC in many cases. Use of both light diffuser fibers was necessary in the present case. The frontal light distributor is easy to handle, whereas the cylindrical light diffuser is difficult to manipulate under ERC. Laser light must be delivered to the tumor lesion through an endoscope. However, as the light diffuser fiber structures are very thin, they cannot be passed through the bent part of the endoscope during endoscopic retrograde cholangio pancreatography (ERCP). Some devices that use a cylindrical light diffuser during ERC are available. We inserted the diffuser into a 10-Fr. sheath of a plastic stent delivery system (MAJ1422; Olympus, Tokyo, Japan) as a preloaded catheter. Moreover, we placed these devices in an adequate position blindly using a transpapillary approach because the 10-Fr. preloaded sheath and cylindrical light diffuser have no radiopaque markers. Accordingly, we confirmed the position of the device tip translucently using an X-ray fluoroscopy contrast medium. We used a Y-connector (GateWay ${ }^{\circledR}$ PLUS YAdapter; Boston Scientific, Tokyo, Japan) at the opposite side of the 10-Fr. preloaded sheath tip to adjust the placement of the devices, with simultaneous injection of the contrast medium. We treated this patient appropriately using a cylindrical light diffuser and these devices under ERC. However, improvements in these devices are still needed in the future.

In summary, we herein described a case of PDT using talaporfin sodium and a PD laser ${ }^{\circledR}$ to treat recurrent cholangiocarcinoma after surgical resection. Few cases have reported using talaporfin sodium as a photosensitizer for cholangiocarcinoma and the subsequent endoscopic findings during PDT follow-up for cholangiocarcinoma. We confirmed no recurrence of cholangiocarcinoma during this follow-up period based on the endoscopic, cholangiographic, and pathological findings after PDT. Furthermore, no adverse effects were observed following this procedure. Talaporfin sodium has a shorter sun-shade period than porfimer sodium, and illumination with the PD $\operatorname{laser}^{\circledR}$ is considerably easier than using the excimer dye laser system. PDT using talaporfin sodium and the PD laser ${ }^{\circledR}$ for cholangiocarcinoma may be a useful option as an active, curative, and safe treatment. Further investigation with a larger number of cases is necessary to clarify the effectiveness and safety of this procedure.

The authors state that they have no Conflict of Interest (COI).

\section{References}

1. Kiesslich T, Wolkersdorfer G, Neureiter D, Salmhofer H, Berr F. Photodynamic therapy for non-resectable perihilar cholangiocarcinoma. Photochem Photobiol Sci 8: 23-30, 2009.

2. Nanashima A, Abo T, Nonaka T, et al. Photodynamic therapy using talaporfin sodium (Laserphyrin(R)) for bile duct carcinoma: a preliminary clinical trial. Anticancer Res 32: 4931-4938, 2012.

3. Kahaleh M, Mishra R, Shami VM, et al. Unresectable cholangiocarcinoma: comparison of survival in biliary stenting alone versus stenting with photodynamic therapy. Clin Gastroenterol Hepatol 6: 290-297, 2008.

4. Ortner ME, Caca K, Berr F, et al. Successful photodynamic therapy for nonresectable cholangiocarcinoma: a randomized prospective study. Gastroenterology 125: 1355-1363, 2003.

5. Prasad GA, Wang KK, Baron TH, et al. Factors associated with increased survival after photodynamic therapy for cholangiocarcinoma. Clin Gastroenterol Hepatol 5: 743-748, 2007.

6. Park do H, Lee SS, Park SE, et al. Randomised phase II trial of photodynamic therapy plus oral fluoropyrimidine, S-1, versus photodynamic therapy alone for unresectable hilar cholangiocarcinoma. Eur J Cancer 50: 1259-1268, 2014.

7. Witzigmann H, Berr F, Ringel U, et al. Surgical and palliative management and outcome in 184 patients with hilar cholangiocarcinoma: palliative photodynamic therapy plus stenting is comparable to r1/r2 resection. Ann Surg 244: 230-239, 2006.

8. Wiedmann M, Berr F, Schiefke I, et al. Photodynamic therapy in patients with non-resectable hilar cholangiocarcinoma: 5-year follow-up of a prospective phase II study. Gastrointest Endosc 60: 68-75, 2004.

9. Nanashima A, Yamaguchi H, Shibasaki S, et al. Adjuvant photodynamic therapy for bile duct carcinoma after surgery: a preliminary study. J Gastroenterol 39: 1095-1101, 2004.

10. Wiedmann M, Caca K, Berr F, et al. Neoadjuvant photodynamic therapy as a new approach to treating hilar cholangiocarcinoma: a phase II pilot study. Cancer 97: 2783-2790, 2003.

11. Cheon YK. The role of photodynamic therapy for hilar cholangiocarcinoma. Korean J Intern Med 25: 345-352, 2010.

12. Nanashima A, Isomoto $H$, Abo $T$, et al. How to access photodynamic therapy for bile duct carcinoma. Ann Transl Med 2: 23, 2014.

13. Ortner MA. Photodynamic therapy for cholangiocarcinoma: overview and new developments. Curr Opin Gastroenterol 25: 472476, 2009.

14. Yano T, Muto M, Yoshimura K, et al. Phase I study of photodynamic therapy using talaporfin sodium and diode laser for local failure after chemoradiotherapy for esophageal cancer. Radiat On- 
Intern Med 54: 2321-2326, 2015 DOI: 10.2169/internalmedicine.54.4673

col 7: 113, 2012.

15. Igami T, Nagino M, Oda K, et al. Clinicopathologic study of cholangiocarcinoma with superficial spread. Ann Surg 249: 296-302, 2009.
16. Gao F, Bai Y, Ma SR, Liu F, Li ZS. Systematic review: photodynamic therapy for unresectable cholangiocarcinoma. J Hepatobiliary Pancreat Sci 17: 125-131, 2010.

(C) 2015 The Japanese Society of Internal Medicine http://www.naika.or.jp/imonline/index.html 